\title{
An Inductance-Based Sensing System for Bellows-Driven Continuum Joints in Soft Robots
}

\author{
Wyatt Felt*, Maria J. Telleria ${ }^{\dagger}$, Thomas F. Allen ${ }^{\dagger}$, Gabriel Hein ${ }^{\dagger}$, Jonathan B. Pompa ${ }^{\dagger}$, Kevin Albert ${ }^{\dagger}$, C. David Remy* \\ * Robotics and Motion Laboratory (RAMlab), University of Michigan, Ann Arbor, MI. Emails: (wfelt, cdremy)@umich.edu \\ $\dagger$ Pneubotics, an Otherlab Company, San Fransisco, CA. Email: info@pneubotics.com
}

\begin{abstract}
In this work we present a novel, inductance-based system to measure and control the motion of bellows-driven continuum joints in soft robots. The sensing system relies on coils of wire wrapped around the minor diameters of each bellows on the joint. As the bellows extend, these coils of wire become more distant, decreasing their mutual inductance. Measuring this change in mutual inductance allows us to measure the motion of the joint. By dividing the sensing of the joint into two sections and measuring the motion of each section independently, we are able to measure the overall deformation of the joint with a piecewise constant-curvature approximation. This technique allows us to measure lateral displacements that would be otherwise unobservable. When measuring bending, the inductance sensors measured the joint orientation with an RMS error of $1.1^{\circ}$. The inductance sensors were also successfully used as feedback to control the orientation of the joint. The sensors proposed and tested in this work provided accurate motion feedback that would be difficult to achieve robustly with other sensors. This sensing system enables the creation of robust, self-sensing, and soft robots based on bellows-driven continuum joints.
\end{abstract}

\section{INTRODUCTION}

The emerging field of soft robotics is enabling fundamentally new ways to design, build, and control robotic systems. Such soft robots exhibit complex behaviors that emerge from deliberate compliance in the actuators and structure. By incorporating passive degrees of freedom into their structure, soft robots can passively conform to the constraints of their environment and to the objects they are manipulating. Many soft robots are actuated by the flexible expansion of hermetically sealed volumes driving compliant joints. Systems based on these principles are lightweight, flexible and have low reflected inertia. This makes them inherently safe in physical human robot interaction. Moreover, the sealed actuators and flexible joints are well-suited to work in harsh environments where external contaminates could breach the dynamic seals of rotating or sliding shafts.

In this paper, we focus on sensing and controlling the motion of bellows-driven continuum joints. The sensing system relies on coils of insulated conductive wire wrapped around the minor diameters of the bellows. These coils form circuits with inductance values that change with the length of the bellows. The measured inductance values can be calibrated to measure the motion of the joint. We demonstrate experimentally how sensors such as these can measure and control the motion of bellows-driven continuum joints.

We utilize a joint made from four pneumatically driven

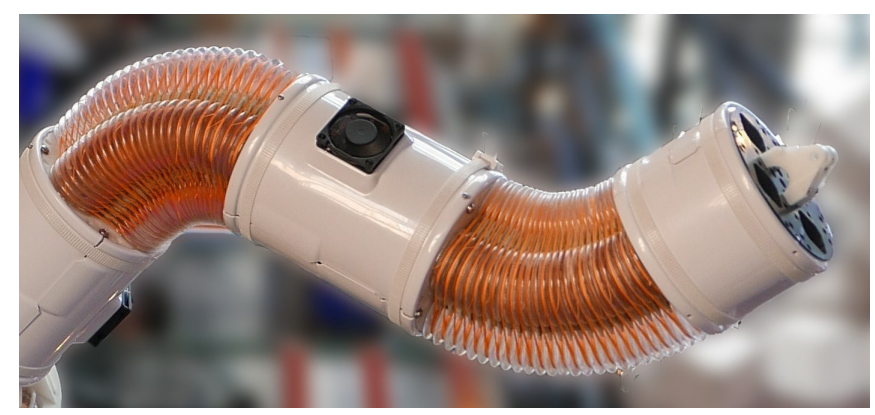

Fig. 1. Bellows-driven continuum joints are used to create robots without finite degrees of freedom. Sensing the motion of such robots is a challenge. The inductance-based sensors presented in this work will bring estimation and control to robots like the one pictured here (created by Pneubotics).

bellows that are positioned around a central steel cable (Fig. 1). This joint has been developed by Pneubotics. The bellows create bending torques about two axes while keeping the joint stiff in torsion. By pressurizing pairs of antagonized bellows simultaneously, the passive bending stiffness of the joint is also controllable. Joints with similar features have been developed for applications in industry and academia. These have relied on bellows [10, 3, 15, 12, 11] and other soft, fluid-powered actuators [5, 18, 16].

The advantages of soft continuum joints come with the new challenge of sensing and controlling their distributed motion. While traditional robotic systems provide discrete mechanical joints on which to couple rotational or translational sensors, soft continuum joints, by their nature, do not have such convenient coupling points. Instead, the deformation of soft joints is typically measured with an external localization system, inertial measurement units (IMUs), or a set of internal sensors.

External localization systems include visual "3D motion capture," electromagnetic tracking, and radio frequency indoor positioning systems. Visual localization systems typically rely on retro-reflective markers or laser beacons [31, 22]. These systems require a line-of-sight to operate. Accordingly, they have limited utility in visually occluded workspaces. Electromagnetic tracking systems [29, 23] avoid these occlusion problems but have smaller workspaces. Radio Frequency systems may have vast workspaces but limited accuracy [30]. Moreover, high-frequency signals rapidly attenuate underwater.

In some situations, IMUs can be used to estimate the motion 
of difficult-to-sense joints in robots and humans [13, 4]. Orientation estimates from IMUs, however, are not always accurate. Without reliable magnetic field information, IMU heading estimates are susceptible to drift. Naturally, orientation estimates can only measure robot motion that changes orientation. This is not always the case. Continuum joints, for example, can deflect laterally without changing the relative orientation of the joint ends.

Internal sensors measure the deformation in the system directly. The deformation can be measured, for example, by recording changes in length along well-defined paths. The length of joint sections, for example, can be measured through the recoil of strings or tendons under tension [16, 10]. Strain can also be measured using elastomers with elements that exhibit changes in resistance or capacitance [2, 24, 26]. Optical fibers can measure strain (and thus bending) via Fiber Bragg Gratings [17] or deformation-induced attenuation [25]. Elastomeric waveguides can also be used [32, 28]. The shape of cable-like sensors can be measured through the changing distance between pairs of LEDs and phototransistors [14, 11]. Inductance-based sensors have been developed by the authors Felt and Remy for use in soft actuators such as bending bellows [8] and McKibben muscles [7, 9, 6].

Among these sensing technologies, inductance-based sensors provide unique advantages. String recoil systems are often bulky and fragile. Elastomeric and optical fiber systems can fail under repeated strain and may require specialized equipment to fabricate. Inductance-based systems rely on offthe-shelf, high-flex-life wire to create inexpensive and lowprofile sensors.

The primary contribution of this work is the introduction of inductance sensors that measure the motion of bellowsdriven continuum joints. We develop the theory, models and design principles for these sensors. The experimental sensing system measures the motion of the joint independently in two halves along the joint length. This allows us to measure lateral displacement even when there is no change in orientation between the ends. The performance of the sensing system is tested in both quasi-static conditions and as feedback for the control of a bellows-driven joint.

The hardware of our experimental system is described in Section III Section III discusses theory, including the kinematics of the joint (III-A), models for the inductance sensor (III-B) and design principles relating to the same (III-C). Section III-D investigates the use of "split-joint" sensing to measure lateral displacement. Our experimental methods and results are described in Section IV, This includes the calibration and verification of the sensing system (IV-A), the estimation of the joint position under lateral loads (IV-B) and the feedback control of the joint orientation (IV-C). This is followed by a general discussion in Section $\mathrm{V}$.

\section{HARDWARE}

Our inductance-based sensing system was implemented on a commercial, bellows-driven continuum joint. To create a selfsensing joint based on inductance, the minor diameters of the bellows were wrapped with flexible wire (Fig. 2). This created circuits of circular coils spaced along the length of the bellows. As a bellows expanded in length, the circular coils moved farther apart, reducing the inductance of the corresponding circuit. The joint was instrumented and controlled to calibrate and test the inductance-based sensing system.

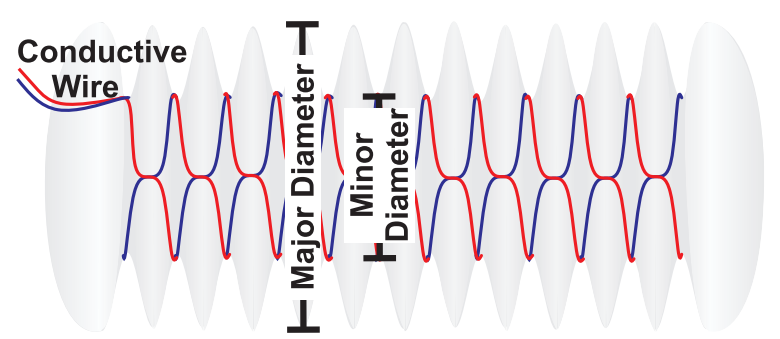

Fig. 2. The minor diameters of the plastic bellows were wrapped with insulated conductive wire (red and blue). The inductance of the circuit provides a measure of the bellows length.

The joint was provided by Pneubotics (an Otherlab company, San Fransico, CA, USA, Fig. 11. The joint consists of two plates connected to four bellows spaced around a central steel cable. The centers of the bellows are kept at a fixed distance, designated $b$, of $4.9 \mathrm{~cm}$ from the central cable. The steel cable has a length $h$ of $19.7 \mathrm{~cm}$ between the plates of the joint. It provides a "fulcrum" to convert the extension forces of the bellows into bending moments. The bellows have 26 major diameters between the plates of the joint. The major and minor diameters of the bellows are $6.7 \mathrm{~cm}$ and $4.9 \mathrm{~cm}$, respectively. The joint is actuated by pressurizing the bellows with compressed air. The antagonized configuration of the four bellows creates a 2-DOF bending joint with independently controllable joint torque and passive stiffness. The unmodified joint has a range of motion of $\pm 90^{\circ}$ in each axis. In this work, the pressure in the bellows was maintained below $0.41 \mathrm{MPa}$.

The joint was outfitted with four distinct inductance circuits (Fig. 3). Pairs of adjacent circuits measured the bending in each half of the joint. The circuits were formed from "tinsel" wire with a high flex-fatigue life (TN3637, $1.14 \mathrm{~mm}$ outer diameter, resistance 538 ohms $/ \mathrm{km}$, MN wire, St. Paul, MN, USA). The flexible wire was wrapped around 12 minor diameters of the bellows in the corresponding half. Each minor diameter had two turns of current (except at the ends of the circuits where there was only one turn). The inductance was measured with an LDC1614 chip (Excitation voltage: 1.2-1.8 V, Texas Instruments, Dallas, TX, USA). This chip measures the resonant frequency of four inductor-capacitor oscillating circuits in rapid succession. To this end, each inductive circuit was connected in parallel with a high-precision $(1 \%, \mathrm{NP} 0) 100 \mathrm{pF}$ ceramic capacitor.

To provide a ground truth reference for our sensor, the joint was mounted upside-down on a level mount such that the relative orientation of the ends could be measured with an IMU (Dynamic accuracy: $\pm 1^{\circ}, 3$-Space Micro USB, magnetometer disabled, Yost Labs, Portsmouth, OH, USA). A $38 \mathrm{~cm}$ arm was attached to the end of the joint for calibration and testing. 

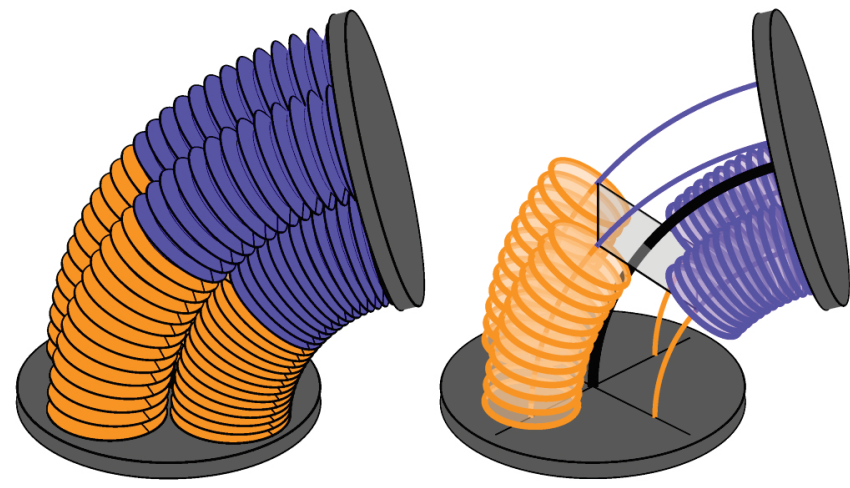

Fig. 3. The deformation of the entire joint was approximated as the composition of two constant-curvature sections. Pairs of adjacent inductive sensor circuits (orange and blue circles) measured the bending of the each half independently. This "split-joint" configuration allows us to estimate the joint motion in non-uniform-curvature conditions.

Weights were added to the end of the arm to create different loading conditions. The pressure in the bellows was controlled with electronic pressure regulators (TR, Enfield Technologies, Shelton, CT, USA). The data acquisition and control was facilitated by LabVIEW.

\section{THEORY}

\section{A. Kinematic Model}

The joint was modeled as the composition of two constant curvature sections (Fig. 3). This was designed to allow the deformation to be approximated even when the curvature across the length of the joint is not uniform.

For each constant-curvature section of the joint, the coordinate axes in the base frame originate at the center of the central cable and intersect with the bellows' centers (Fig. 4). The xaxis points towards bellows 1 , the y-axis towards bellows 2 , and the $\mathrm{z}$-axis along the central cable (when straight). The bellows' centers are separated from the central cable by the constant distance $b$.

We describe the kinematics of each constant curvature section joint using a parametrization presented by Allen, et al. [1]. This parametrization has several desirable properties. It remains invertible in the straight configuration and has affine relationships between the rotation parameters and the lengths of the bellows. This parametrization is based on the two components, $u$ and $v$, of a rotation vector $\boldsymbol{\omega}=[u, v, 0]^{T}$. The $\mathrm{z}$-component is always zero. This rotation vector $\boldsymbol{\omega}$ describes the orientation of the top of the plate relative to the base and is equivalent to rotating the top plate by an angle $\theta=\sqrt{u^{2}+v^{2}}$ around the unit vector $\boldsymbol{\omega} /\|\boldsymbol{\omega}\|$. The rotation vector $\boldsymbol{\omega}$ can also be described by the angles $\phi$ and $\theta$

$$
\boldsymbol{\omega}=[u, v, 0]^{T}=[-\theta \sin \phi, \theta \cos \phi, 0]^{T} .
$$

The homogeneous transformation from the base frame to a frame with distance $h$ along the cable (assuming constant curvature across that distance) is given by the matrix
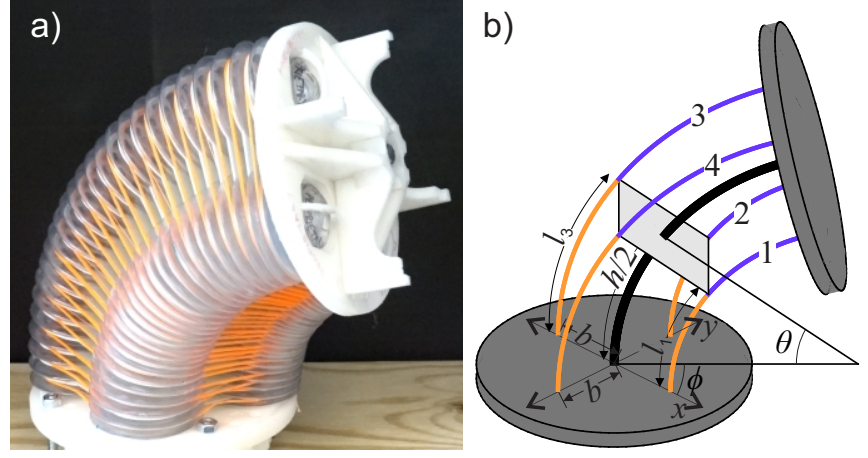

Fig. 4. (a) Photo of a 2-DOF bellows-driven continuum joint. The orange fibers constrain the bellows around the central cable. (b) The kinematic model of the joint. Each half of the joint undergoes a bend angle $\theta$ with an orientation $\phi$. The center of the joint is reinforced by a cable of length $h$ (thick black line). The bellows are indexed from one to four. The center-lines of the half bellows (thin blue and orange lines) have lengths of $l_{1}, l_{2}, l_{3}$ and $l_{4}$. The centers of the bellows are spaced from the central cable by a distance $b$.

$\mathbf{g}(u, v, h)$

$$
\mathbf{g}(u, v, h)=\left[\begin{array}{cccc}
\gamma v^{2}+1 & -\gamma u v & \zeta v & -\gamma h v \\
-\gamma u v & \gamma u^{2}+1 & -\zeta u & \gamma h u \\
-\zeta v & \zeta u & \cos (\theta) & \zeta h \\
0 & 0 & 0 & 1
\end{array}\right]
$$

The functions $\zeta(\theta)=\sin (\theta) / \theta$ and $\gamma(\theta)=(\cos (\theta)-1) / \theta^{2}$ are defined when $\theta$ is zero. This is apparent from the Maclaurin series of sine and cosine.

The lengths $l=f(u, v)$ of the half-bellows sections along their center-lines are expressed as follows:

$$
\left[l_{1}, l_{2}, l_{3}, l_{4}\right]^{T}=\frac{h}{2}+b[-v, u, v,-u]^{T} .
$$

Because $h$ is fixed, the length $l$ of each bellows section is a function of either only $v$ or only $u$.

The bending in the distal half of the joint was defined by $\boldsymbol{\omega}_{a}=\left[u_{a}, v_{a}, 0\right]^{T}$ and measured by the inductance values on the distal halves of bellows 1 and 2 (Fig. 3, blue). The proximal joint half was defined by $\boldsymbol{\omega}_{b}=\left[u_{b}, v_{b}, 0\right]^{T}$ and measured with inductance sensors on bellows 3 and 4 (Fig. 3, orange).

\section{B. Inductance Model}

The inductive circuits are modeled as $n$ circular coils of current connected electrically in series. Each circular coil is made up of $N$ turns of wire. The total inductance $L$ of the circuit is the sum of the self-inductance $L_{i, i}^{\prime}$ and mutual inductance $M_{i, j}$ of the coils in the circuit. The total inductance $L=\sum_{i=1}^{n} \sum_{j=1}^{n} \mathbf{L}[i, j]$ is the sum of the elements in the inductance matrix $\mathbf{L}$

$$
\mathbf{L}=\left[\begin{array}{ccccc}
L_{1,1}^{\prime} & M_{1,2} & M_{1,3} & \ldots & M_{1, n} \\
M_{2,1} & L_{2,2}^{\prime} & M_{2,3} & \ldots & M_{2, n} \\
M_{3,1} & M_{3,2} & L_{3,3}^{\prime} & \ldots & M_{3, n} \\
\vdots & \vdots & \vdots & \ddots & \vdots \\
M_{n, 1} & M_{n, 2} & M_{n, 3} & \ldots & L_{n, n}^{\prime}
\end{array}\right]
$$


The self-inductance of the individual coils $L_{i, i}^{\prime}$ does not change during actuation. A circular wire coil with $N$ turns of current, a coil radius $r$ and a wire radius $a$ has a selfinductance that is approximated by

$$
L_{i, i}^{\prime} \approx \mu N^{2} r\left(\ln \left(\frac{8 r}{a}\right)-2\right) .
$$

This approximation assumes that the current distribution is concentrated on the surface of the conductors. $\mu$ is the magnetic permeability of the surrounding medium (approximately $4 \pi \times 10^{-7} \mathrm{H} / \mathrm{m}$ for nonmagnetic materials such as plastic and air).

The sensitivity of the inductance to joint motion comes from the change in mutual inductance between coils on different minor diameters. For these current paths, the mutual inductance is calculated numerically by integrating the Neumann formula [19]. For two paths in 3D space

$$
\begin{aligned}
& \boldsymbol{C}_{\mathbf{1}}\left(s_{1}\right)=\left[x_{1}\left(s_{1}\right), y_{1}\left(s_{1}\right), z_{1}\left(s_{1}\right)\right]^{T} \\
& \boldsymbol{C}_{\mathbf{2}}\left(s_{2}\right)=\left[x_{2}\left(s_{2}\right), y_{2}\left(s_{2}\right), z_{2}\left(s_{2}\right)\right]^{T}
\end{aligned}
$$

parameterized by $s_{1}=[0,1], s_{2}=[0,1]$, the mutual inductance is given the double integral

$$
M_{1,2}=\frac{\mu}{4 \pi} \int_{0}^{1} \int_{0}^{1} \frac{\left(\left.\frac{d \boldsymbol{C}_{\mathbf{1}}}{d s_{1}}\right|_{s_{1}}\right)\left(\left.\frac{d \boldsymbol{C}_{\mathbf{2}}}{d s_{2}}\right|_{s_{2}}\right)^{T}}{\sqrt{\left(\boldsymbol{C}_{\mathbf{1}}-\boldsymbol{C}_{\mathbf{2}}\right)\left(\boldsymbol{C}_{\mathbf{1}}-\boldsymbol{C}_{\mathbf{2}}\right)^{T}}} d s_{1} d s_{2} .
$$

The mutual inductance between two $N$-turn coils on separate convolutions was approximated as $N^{2}$ times the mutual inductance between single-turn coils (circular loops) on the minor diameters of the bellows. This approximation is accurate when the distance between the turns in each coil is small relative to the distance between the two coils.

The inductance values of the circuits on the bellows change with the deformation of their corresponding joint section. For example, the inductance of a sensor on bellows 1 in the proximal half of the joint, $L_{1 a}=f\left(u_{a}, v_{a}\right)$, is a function of the curvature of the joint in that half. In order to measure the motion of the joint, we desire to invert this relationship (e.g. $\left.u_{a}=f\left(L_{1 a}, L_{2 a}, L_{3 a}, L_{4 a}\right)\right)$. We used the kinematic and inductance models to investigate which combinations of inductance sensors are suitable for use in this inversion. To this end, Eq. (7) was used to calculate the inductance of the circuits at different joint orientations. A circular loop of current was first defined as a geometric path. This path was then transformed by Eq. (2) to the appropriate positions around the joint as it underwent constant-curvature bending. For each pair of circular loops in a circuit, Eq. (7) was integrated with the MATLAB integral2 function. To examine the effect of sensor placement, the sensors were modeled to be on the same section of the joint (i.e. with geometries dependent on $u_{a}$ and $v_{a}$ ). In this configuration, the length change of the sensor modeled on bellows 1 was equal and opposite of that on bellows 3 . The same relationship holds for bellows 2 and 4 .

The geometry and corresponding inductance values were calculated at each combination of a series of 22 values of $\phi$ and 12 values of $\theta$. The values of $\phi$ were equally spaced between $0^{\circ}$ and $343.64^{\circ}$ and the values of $\theta$ where equally spaced between $0^{\circ}$ and $90^{\circ}$. The inductance values were calculated only once when $\theta=0$ (where $\phi$ does not change the geometry).

The modeled inductance values were used to evaluate four different sensor combinations. The first was a single-variable fourth-order polynomial regression of the length-changing rotation component against the modeled inductance of the sensor on bellows $1\left(v_{a}=f\left(L_{1 a}\right)\right)$. The second regression was against the difference of the modeled inductance of the sensors on the antagonized pair, bellows 1 and $3\left(v_{a}=\right.$ $\left.f\left(L_{1 a}-L_{3 a}\right)\right)$. The third combination was a two-variable polynomial regression against the adjacent sensors on bellows 1 and $2\left(v_{a}=f\left(L_{1 a}, L_{2 a}\right)\right)$. The final combination was a twovariable regression on the differences of each antagonized pair $\left(v_{a}=f\left(L_{1 a}-L_{3 a}, L_{2 a}-L_{4 a}\right)\right)$. The residual error of these regression types is listed in Table $\mathrm{I}$

TABLE I

Model Estimates of $v_{a}$ WITH COMBINATIONS OF SENSORS

\begin{tabular}{|l||c|c|}
\hline Polynomial Type $(4$ th Order) & RMSE $\left(^{\circ}\right)$ & $R^{2}$ \\
\hline \hline$v_{a}=f\left(L_{1 a}\right)$ & 0.323 & 0.9997286 \\
$v_{a}=f\left(L_{1 a}-L_{3 a}\right)$ & 0.149 & 0.9999421 \\
$v_{a}=f\left(L_{1 a}, L_{2 a}\right)$ & 0.029 & 0.9999979 \\
$v_{a}=f\left(L_{1 a}-L_{3 a}, L_{2 a}-L_{4 a}\right)$ & 0.020 & 0.9999990 \\
\hline
\end{tabular}

The single-variable regression $\left(v_{a}=f\left(L_{1 a}\right)\right)$ explained $99.97 \%$ of the variation in the corresponding rotation component (Fig. 5). The bulk of the remaining error comes from the variance introduced by the orthogonal rotation component (e.g. $u_{a}$ ). The second combination looked at the difference between the inductance values $L_{1 a}$ and $L_{3 a}$. If $u_{a}$ were to effect $L_{1 a}$ and $L_{3 a}$ identically, the effect of $u_{a}$ would be canceled in the difference. Though the effect of $u_{a}$ on the two sensors is not identical, the regression against $L_{1 a}-L_{3 a}$ did lower the RMSE by $54 \%\left(v_{a}=f\left(L_{1 a}-L_{3 a}\right)\right)$. Including measurements from a sensor that primarily measures $u_{a}\left(v_{a}=f\left(L_{1 a}, L_{2 a}\right)\right)$ reduced the RMSE by an order of magnitude (compared to the single variable regression). A regression against the differences of both antagonized pairs only reduced the RMSE by an additional $30 \%$. The strategy of using two adjacent sensors on the same joint section (e.g. $\left.v_{a}=f\left(L_{1 a}, L_{2 a}\right)\right)$ is adopted experimentally in this work.

\section{Design Principles for Inductance Sensors on Bellows}

Bellows-based inductance sensors exhibit the greatest sensitivity to motion when the minor diameters of the bellows are close together relative to the size of the diameters. Consider two coaxial circular wire coils of a single turn separated by a distance $h^{\prime}$ along their mutual axis. If the coils are moved closer together, the mutual inductance between them increases.

The change in mutual inductance per distance traveled is also affected by the distance between the coils. The sensitivity of the mutual inductance to a change in distance is $\frac{d M}{d h^{\prime}}$. Its magnitude is greatest when the coils are close together and declines rapidly as they move farther apart. For example, from 


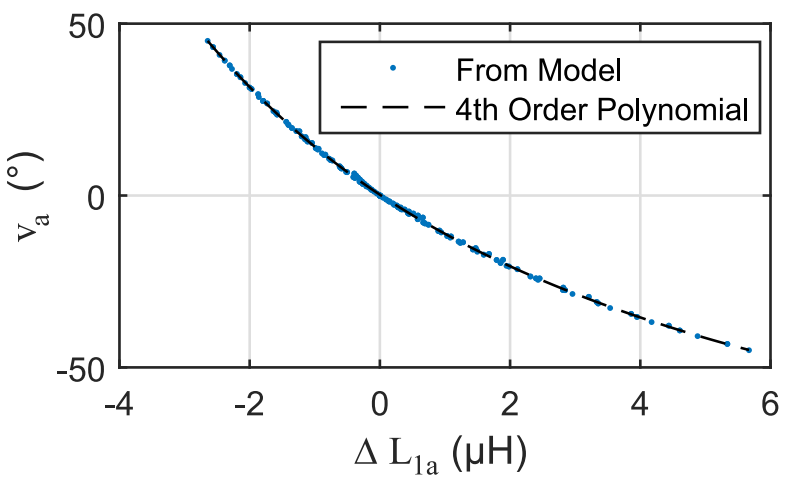

Fig. 5. The results of the inductance model for the joint used in this work. Much of the variation in the rotational components (e.g. $v_{a}$ ) is explained by a simple polynomial regression against the inductance of a coil on the length-changing bellows (e.g. $L_{1 a}$ ).

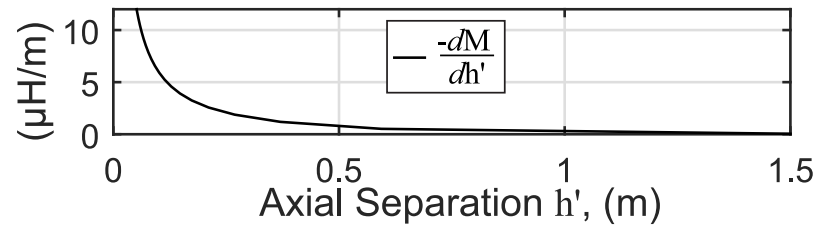

Fig. 6. Shown is the mutual inductance sensitivity to separation of two coaxial circles of equal diameter $(1 \mathrm{~m})$ separated by a distance $h^{\prime}$. The change in mutual inductance per distance traveled declines rapidly as the coils move farther apart.

an axial distance of $h^{\prime}=.05$ diameters to $h^{\prime}=1$ diameter, the sensitivity decreases by approximately two orders of magnitude (Fig. 6).

Thus inductance sensors are best-suited to work on bellows with minor diameters that are spaced much more closely than the size of the diameters themselves. The bellows used in this work, for example, have $h^{\prime}$ values of approximately 0.014 diameters when the joint is straight.

Another consideration is how many turns of wire to use in each coil. This consideration has trade-offs in sensor quality and actuation range. One measure of the quality of an inductance sensor is the "Quality Factor" $Q$

$$
Q=2 \pi f_{\text {excite }} \frac{L}{R}
$$

where $R$ is the resistance, $L$ the inductance, and $f_{\text {excite }}$ the excitation frequency. The maximum excitation frequency is often limited by the sensing circuitry or parasitic capacitance [21]. Thus, for a given frequency, it is desirable to maximize the ratio of inductance to resistance. The inductance scales with the radius $r$ of the coils and with the square of the number of turns $N^{2}$ in each coil

$$
L \propto r N^{2} .
$$

The resistance is proportional to the number of turns $N$ and the radius $r$ of the circular coils and inversely proportional to the cross-sectional area of the conductor $A_{\text {wire }}$

$$
R \propto \frac{N r}{A_{\text {wire }}} .
$$

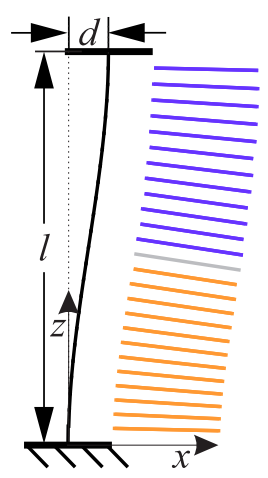

Fig. 7. The geometry of the joint was simulated with a level displacement $d$. Our models predict that using two circuits along the length of the joint improves the estimation of deformations like these.

Accordingly, the inductance to resistance ratio scales linearly with the number of turns $N$ and the cross-sectional area of the conductors $A_{\text {wire }}$

$$
\frac{L}{R} \propto N A_{\text {wire }}
$$

Thus, increasing the number of turns in each coil or increasing the cross-sectional area of the conductors increases the sensor quality. However, there are trade-offs to increasing these quantities. Increasing the number of turns can increase the parasitic capacitance which, if it becomes too high, can lower the feasible excitation frequency [21]. Furthermore, the wires take up physical space on the minor diameters. The cross-sectional area $A_{\text {coil }}$ of the circular coils scales in the same way as $\frac{L}{R}$

$$
A_{\text {coil }} \propto N A_{\text {wire }}
$$

This bulk of material in the convolutions could limit contraction of the bellows. This also suggests that, for a fixed excitation frequency, a high quality factor is more easily achieved with a physically larger system. In this work, the number of turns of wire in each of the circular coils was kept at a minimum.

\section{Measuring Non-uniform Curvature}

When actuated against external loads, the joint may be subject to non-uniform internal bending moments. These may lead to non-uniform curvature along the length of the joint. Measuring the curvature of the joint in multiple sections can improve the ability of the joint to sense certain non-uniformcurvature deformations. To demonstrate how multiple sensing sections can improve the estimation of the joint motion, we simulated the lateral displacement of the joint end with no change in orientation of the plates (Fig. 7). The chosen displacement was selected to highlight the opportunity of using multiple sensors along the joint length.

For this simulation, the profile of the central cable was approximated with the simple planar equations of a thin cantilever. The cantilever we considered has a fixed end and is free but guided at the other end. A force and moment at the free end deflect it a distance $d$ without rotation at the tip. 
The profile of the cantilever with this deflection is given by the following expression [20]

$$
x(z)=\frac{d z^{2}}{l^{3}}(3 l-2 z)
$$

where $z$ is measured from the support along the length of the unloaded beam. $l$ is the distance in $z$ between the ends. $l$ is selected to conserve the length of the central cable.

The geometry of the central cable and coils was calculated for a a lateral displacement of $d=2 \mathrm{~cm}$ in the $x z$-plane along the $x$-axis towards bellows 1 . The profile of the central cable was defined by Eq. (13). The geometric paths describing the circular loops of current were transformed via Eq. (13) to their positions in the displaced configuration (Fig. 7). The mutual inductance between the loops on bellows 1 was then calculated with Eq. (7) and the MATLAB integral2 function. Three different circuit configurations were modeled: one circuit spanning the entire bellows length ( 25 coils), two circuits (12 coils in each half), and three circuits (8-9-8, in each approximate third). The bending in each section was estimated by using the inductance values predicted for the lateral displacement in equations calibrated to constant-curvature bending. A singlevariable, 4th-order polynomial (e.g. $v_{a}=f\left(L_{1 a}\right)$ ) was used for each circuit. The deformation of the total joint was then estimated by composing the curvatures predicted by the calibration equations in each section.

For this type of lateral displacement, using two circuits per length of the joint (compared to one) was predicted to lead to smaller error in the estimates of $d, l$ and $\theta$ (Table II). Three circuits was predicted to further reduce the errors in $d$ and $l$.

TABLE II

MOdEL-Predicted ERRoR IN INDUCTANCE-BASEd ESTIMATES FOR A LATERAL DISPLACEMENT OF $2 \mathrm{CM}$

\begin{tabular}{|l||r|r|r|}
\hline \multicolumn{1}{|c||}{ Variable } & \multicolumn{1}{c|}{1} & \multicolumn{3}{c|}{ Number of Circuits } \\
\hline $\mathrm{d}(\mathrm{mm})$ & -21.01 & -4.88 & \multicolumn{1}{c|}{3} \\
\hline $\mathrm{l}(\mathrm{mm})$ & 1.22 & 0.46 & 0.22 \\
$\theta\left(^{\circ}\right)$ & 0.59 & -0.31 & -0.42 \\
\hline
\end{tabular}

\section{EXPERIMENTAL EVALUATION}

\section{A. Calibration and Verification}

The pressure $P$ in each bellows is given by a base pressure $P_{\text {base }}$ and a relative difference in pressure $\Delta P$ to its antagonized counterpart. The pressure differences $\Delta P_{3}$ and $\Delta P_{2}$ are used because they actuate $v$ and $u$ respectively with a positive sign.

$$
\left[P_{1}, P_{2}, P_{3}, P_{4}\right]^{T}=P_{\text {base }}+\left[-\Delta P_{3}, \Delta P_{2}, \Delta P_{3},-\Delta P_{2}\right]^{T}
$$

The actuators were calibrated using a continuous 11 minute sequence of $\Delta P$ combinations. This resulted in well-distributed combinations of $\Delta P$ values (Fig. 8p). $P_{b a s e}$ was $.2 \mathrm{MPa}$.

The calibration data were concatenated from data collected with each of the following masses attached to the end of the arm (Fig. 8 a): $0 \mathrm{~kg}, 2.3 \mathrm{~kg}, 4.5 \mathrm{~kg}, 6.8 \mathrm{~kg}, 9 \mathrm{~kg}$. The purpose of a)

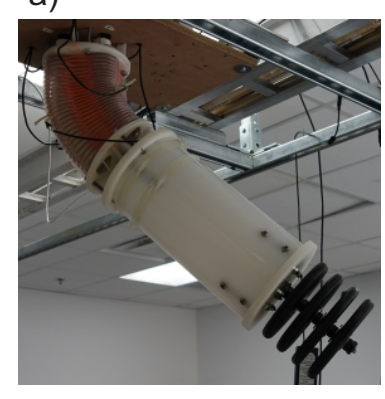

b)

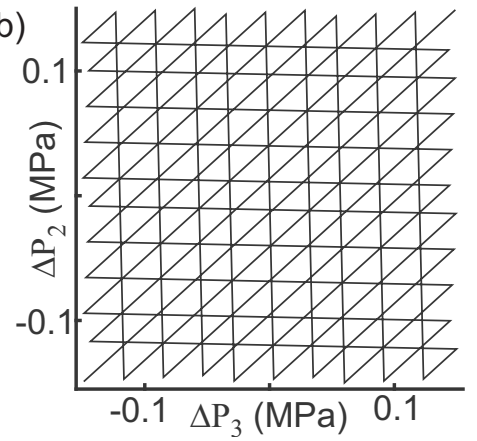

Fig. 8. a) The joint was mounted upside-down on an elevated fixture. Mass was selectively added to the end of the arm for calibration and testing. Shown is a $9 \mathrm{~kg}$ of mass on the end of the arm. b) The combinations of $\Delta P$ used to calibrate the joint.

the added mass was to create a variety of bending conditions for the calibration.

The IMU mounted on the distal plate of the joint provided ground truth measurements of the joint orientation. The IMU measurements were interpreted to find the components of a rotation vector $\hat{\boldsymbol{\omega}}=[\hat{u}, \hat{v}, 0]^{T}$ by assuming the joint deformation to have constant curvature across its entire length. The inductance values from each joint half were regressed with two-variable, 4th order polynomials on $u_{a}=u_{b}=\hat{u} / 2$ and $u_{a}=u_{b}=\hat{v} / 2$.

The calibration was verified against data taken in identical conditions that were not used in the calibration (Fig. 9p. The inductance-predicted orientation of the joint was written as a unit vector in 3-space and compared to the orientation measured by the IMU. An inner product was used to determine the error (measured as a single angle) in the estimated orientation (Table III). As predicted by our inductance models, including the data from the adjacent sensors improved the orientation estimates. Note that 3rd-order, two-variable polynomials resulted in an RMSE of $1.23^{\circ}$ compared to $1.11^{\circ}$ from the 4th-order polynomials.

TABLE III

EXPERIMENTAL VERIFICATION OF JOINT ORIENTATION CALIBRATION

\begin{tabular}{|lll|}
\hline \multicolumn{2}{|c|}{ Polynomial Type (4th Order) } & RMSE ( ${ }^{\circ}$ ) \\
\hline \hline e.g. & $v_{a}=f\left(L_{1 a}\right)$ & $v_{b}=f\left(L_{3 b}\right)$ \\
e.g. $\quad v_{a}=f\left(L_{1 a}, L_{2 a}\right)$ & $v_{b}=f\left(L_{3 b}, L_{4 b}\right)$ & 1.76 \\
\hline
\end{tabular}

\section{B. Estimation of Lateral Displacement}

The purpose of this experiment was to test the ability of the inductance sensors to estimate the end-position of the joint under pure lateral displacement. This type of deformation is unobservable by the IMU. The ground truth in position for this test came from optical markers tracked with an Optitrack V120 Trio camera system (NaturalPoint, Corvalis, OR, USA). The ground truth in orientation came from the IMU. A string tied to the end of the joint was used to deflect the end of the joint towards bellows 1 . The end of the joint was leveled by adjusting the bellows pressures until the IMU reported 


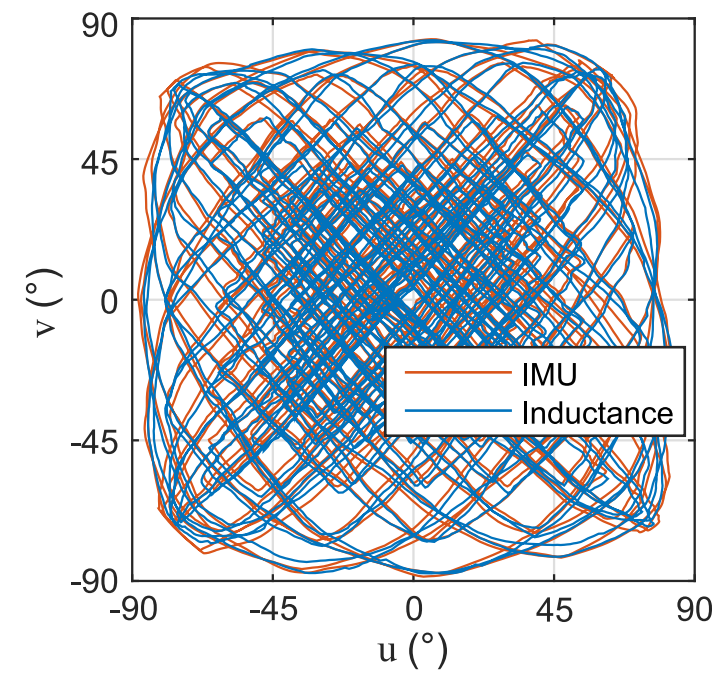

Fig. 9. The rotation components from the verification data set of the joint calibration. The inductance sensors in each half of the joint were calibrated to predict the bending of the joint in that half. Combining the two halves resulted in an overall orientation estimate (blue). This closely matches the orientation measured by the IMU (red).
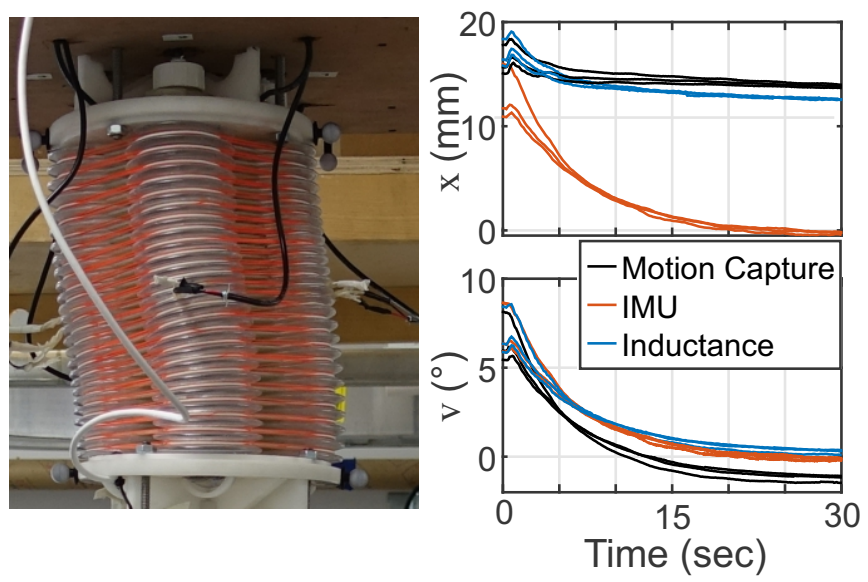

Fig. 10. The photo shows the level joint with a forced, $14 \mathrm{~mm}$ displacement in the direction of bellows $1(x)$. In this condition, the bending in one half of the joint is counteracted by bending in the other half. Also shown are the estimates of the lateral displacement $x$ and the orientation $v$ from the three lateral displacement tests. The lateral displacement predicted by the IMU (red) assumes the joint has a constant curvature across its entire length. As the angle of the joint approaches zero, the IMU displacement estimates (red) also approach zero. The inductance-predicted displacement (blue) remains close to the position recorded by the motion capture system (black). The inductancepredicted estimate of the orientation $v$ also remains close to that measured by the IMU (most accurate).

an approximately level configuration (Fig. 10). The resulting displacement between the ends of the joint was approximately $14 \mathrm{~mm}$. Estimates of the joint displacement $x$ and orientation $v$ were calculated from the measured inductance values and the calibration identified in Section IV-A The test was repeated three times.

From the onset of motion until the final level condition, the inductance provided accurate measures of the joint displacement and orientation (Table IV, Fig. 10p. In the final

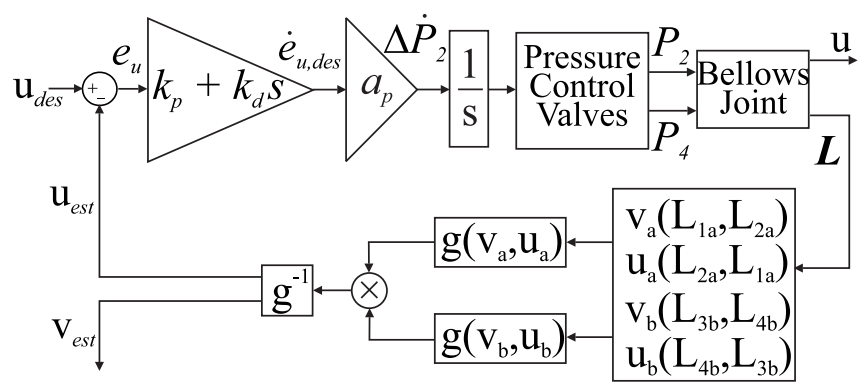

Fig. 11. The feedback controller for the joint relied on the inductancebased estimates of the rotation components $u$ and $v$. The performance of this controller was compared to one driven with feedback from the IMU.

condition, with the joint level and a displacement of $14 \mathrm{~mm}$, the inductance estimate of $v$ had an average error of $0.41^{\circ}$. The inductance estimate of the displacement in $x$ had an average error of $-1.27 \mathrm{~mm}$. The IMU estimate of $x$ had an average error of $-14.1 \mathrm{~mm}$.

TABLE IV

Average RMS of Estimation ERror of Joint Deformation IN THE Lateral Displacement Tests

\begin{tabular}{|l|l||c|c|}
\hline \multirow{2}{*}{ Estimate of } & \multicolumn{1}{|c||}{ Period } & \multicolumn{2}{c|}{ Feedback Type } \\
IMU & Inductance \\
\hline \hline$x(\mathrm{~mm})$ & Entire Test & 12.10 (SD 0.38) & 1.05 (SD 0.19) \\
& Final Condition & 14.1 (SD 0.25) & 1.27 (SD 0.15) \\
\hline$v\left({ }^{\circ}\right)$ & Entire Test & Ground Truth & 0.31 (SD 0.03) \\
& Final Condition & Ground Truth & 0.41 (SD 0.07) \\
\hline
\end{tabular}

\section{Feedback Control}

The inductance sensors were tested in an orientation controller for the components of the rotation vector, $u$ and $v$. The corresponding inputs for these components were $\Delta P_{2}$ and $\Delta P_{3}$, respectively (Eq. (14), $P_{\text {base }}=.2 \mathrm{MPa}$ ). The pressure of the actuators was then controlled with electronic pressure regulators (Fig. 11). The controller gains were scaled by $a_{p}=0.084 \mathrm{MP} / \mathrm{rad} . a_{p}$ is the slope of a line regressed on the calibration data $(0 \mathrm{~kg}$ data only) relating the outputs to the inputs (e.g. $u$ to $\Delta P_{2}$ ). The error $e$ in each rotation component comes from the difference between the reference input $\left(_{\text {des }}\right)$ and the estimated values $(e s t)$

$$
e_{u}=u_{\mathrm{des}}-u_{\mathrm{est}}, e_{v}=v_{\mathrm{des}}-v_{\mathrm{est}} .
$$

The rate-of-change of the commanded pressure differences $\Delta \dot{P}$ depends on this error $e$ and its time derivative $\dot{e}$

$$
\Delta \dot{P}_{2}=a_{p}\left(k_{p} e_{u}+k_{d} \dot{e}_{u}\right), \Delta \dot{P}_{3}=a_{p}\left(k_{p} e_{v}+k_{d} \dot{e}_{v}\right) .
$$

The feedback was tested under two weight conditions $0 \mathrm{~kg}$ $\left(k_{p}=2 \sec ^{-1}, k_{d}=0\right)$ and $9 \mathrm{~kg}\left(k_{p}=2 \sec ^{-1}, k_{d}=0.1\right)$. The estimates of $\dot{e}$ relied on a linear regression over the last 10 data points in time. The loop period of the LabVIEW-based controller was $15 \mathrm{~ms}$. The reference input was a fixed, pseudorandom sequence of ten step changes in combinations of $u$ and $v$. The levels of the steps were chosen to be feasible for the 
given weight condition $\left(60^{\circ}, 30^{\circ}\right.$ and $0^{\circ}$ for $0 \mathrm{~kg} ; 25^{\circ}, 12.5^{\circ}$ and $0^{\circ}$ for $9 \mathrm{~kg}$ ). The steps lasted for ten seconds each. The sequence of steps was repeated three times for each condition. The orientation recorded by the IMU was considered ground truth. For comparison, the controller was also tested with feedback from the IMU (instead of the inductance sensors). The same feedback gains and protocol were used in the IMUcontrolled tests. The performance of the two feedback types was compared with a paired t-test (paired in each step).

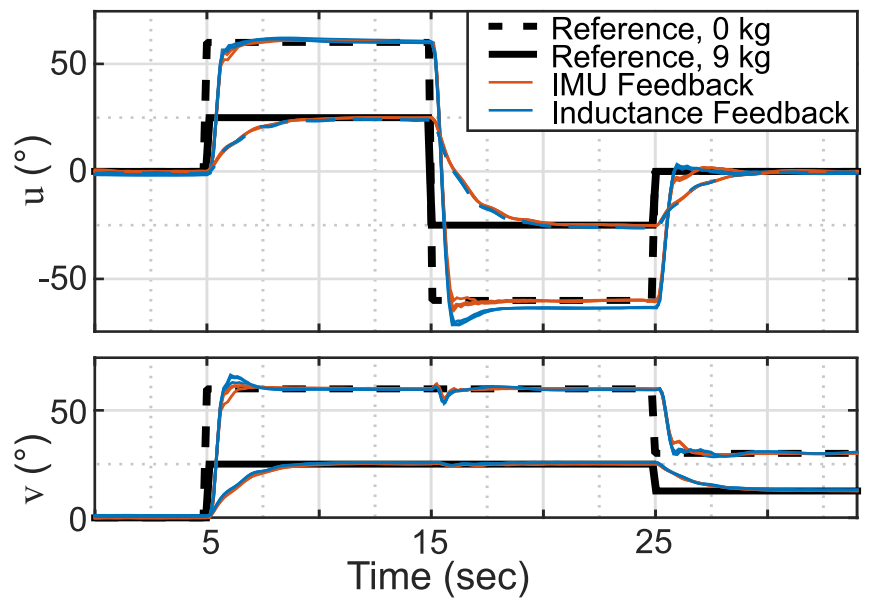

Fig. 12. The data from the early period of the feedback control experiments. The dashed black line is the reference trajectory for the tests in the $0 \mathrm{~kg}$ weight condition. The solid black line corresponds to the $9 \mathrm{~kg}$ tests. The blue lines are the three inductance-feedback tests conducted in each weight condition. The tracking performance of the inductance feedback is comparable to that from the IMU (red lines).

The inductance feedback allowed the joint to track the reference trajectory with similar performance to IMU feedback (Fig. 12, Table V).

TABLE V

AVERAGE RMS OF TRACKING ERROR $\left(^{\circ}\right)$ IN JOINT ORIENTATION FOR EACH REFERENCE STEP OF FEEDbaCK CONTROL TRIALS

\begin{tabular}{|c|c|c|c|c|}
\hline \multirow[b]{2}{*}{ Weight } & \multirow[b]{2}{*}{ Period } & \multicolumn{2}{|c|}{ Feedback Type } & \multirow{2}{*}{$\begin{array}{c}\text { t-test } \\
p\end{array}$} \\
\hline & & IMU & Inductance & \\
\hline \multirow[t]{2}{*}{$0 \mathrm{~kg}$} & First 5 seconds & 13.53 (SD 5.99) & 13.79 (SD 6.02) & $<<0.05$ \\
\hline & Last 5 seconds & 2.48 (SD 1.55) & 2.98 (SD 1.46) & $<0.05$ \\
\hline \multirow[t]{2}{*}{$9 \mathrm{~kg}$} & First 5 seconds & $8.18(\mathrm{SD} 3.70)$ & $8.10(\mathrm{SD} 3.55)$ & $=0.27$ \\
\hline & Last 5 seconds & 1.02 (SD 0.61) & 1.30 (SD 0.55) & $<0.05$ \\
\hline
\end{tabular}

\section{DISCUSSION}

We developed a unique, inductance-based sensing system to measure and control the motion of bellows-driven continuum joints. This system is based on changes in mutual inductance between circular coils on the bellows. Verifying the calibration of our experimental sensing system on a separate data set resulted in an orientation error RMS of only $1.11^{\circ}$ (Fig. 9). In contrast to an IMU, the inductance sensors can measure joint motion that does not change the relative orientation between the ends of a joint. A lateral displacement of $14 \mathrm{~mm}$ was measured by our system with only $1.3 \mathrm{~mm}$ of error. The rapid and accurate inductance measurements enabled a feedback controller to orient a $9 \mathrm{~kg}$ weight on a manipulator arm with a steady-state error of only $1.3^{\circ}$ ( $3^{\circ}$ with no weight). The performance of the inductance-based feedback controller was similar to an IMU-based controller which had a steady-state tracking error which was only $0.5^{\circ}$ and $0.3^{\circ}$ smaller in the respective $0 \mathrm{~kg}$ and $9 \mathrm{~kg}$ conditions.

Future work could lead to additional understanding of inductance sensors for soft robots. The multi-section, constant curvature technique developed in this work could be useful in applying inductance sensing to soft systems driven by bending bellows [8] or fiber-reinforced actuators [7, 9]. Future work could also consider the effect that internal twisting could have on the kinematics and estimation of the joint.

Based on the predictions of the inductance model, each half of our experimental joint relied on only two orthogonal sensors. Using more sensors in a given half, however, could improve the signal-to-noise-ratio. Changes in the rotation component $v_{a}$, for example, create equal and opposite length changes in the corresponding sections of bellows 1 and 3 . Accordingly, the sensitivity of a $v_{a}$ sensor should approximately double when using the difference of $L_{1 a}$ and $L_{3 a}$. Collecting data from redundant sensors on opposite sides of the joint could also allow the system to be accurate even if one side of the joint were in contact with a metal object (which can bias inductance measurements [8, 7]). If multiple inductance-sensing circuits were used in close proximity (e.g. on separate halves of the same bellows), active strategies could be necessary to prevent cross-talk [27].

The inductance-based sensors developed in this work bring sensing and control to otherwise difficult-to-sense continuum joints. Unlike discrete joints, continuum joints have no fixed center of rotation on which to affix an encoder. Alternative sensors proposed for continuum joints are often fragile or otherwise poorly-suited for harsh, real-world applications.

Self-sensing, bellows-driven continuum joints will enable robots that can create and control compliant yet forceful motions in harsh environments. These unique structures will provide inherently compliant actuation without backlash or stiction. Both the sensors and actuators will be made from lightweight and low-cost components. The flexible structure of the continuum joint will allow the robots to conform to external constraints. The absence of discrete mechanical joints in sensors or actuators will allow them to work in harsh environments where sliding surfaces would be vulnerable. The sensing technology developed in this work provides a critical step towards the full implementation of such robotic systems.

\section{ACKNOWLEDGMENTS}

This material is based upon work supported by the National Science Foundation Graduate Research Fellowship under Grant No. DGE 1256260. Any opinion, findings, and conclusions or recommendations expressed in this material are those of the author(s) and do not necessarily reflect the views of the National Science Foundation. Funding for this project was provided by NIH (GRANT: 1-R01-EB0198342014 Wearable eMbots to Induce Recovery of Function). 


\section{REFERENCES}

[1] Thomas F. Allen, Gabriel Hein, and Kevin Albert. Constant-curvature continuum manipulator kinematics. Under Review.

[2] Morteza Amjadi, Ki-Uk Kyung, Inkyu Park, and Metin Sitti. Stretchable, skin-mountable, and wearable strain sensors and their potential applications: A review. Advanced Functional Materials, 2016.

[3] Keith Antonelli and Guy Immega. An extensible robotic tentacle. Industrial Robot: An International Journal, 24 (6):423-427, 1997. doi: 10.1108/01439919710192545. URL http://dx.doi.org/10.1108/01439919710192545

[4] E. R. Bachmann, I. Duman, U. Y. Usta, R. B. McGhee, X. P. Yun, and M. J. Zyda. Orientation tracking for humans and robots using inertial sensors. In Computational Intelligence in Robotics and Automation, 1999. CIRA '99. Proceedings. 1999 IEEE International Symposium on, pages 187-194, 1999. doi: 10.1109/CIRA.1999. 810047.

[5] M. Cianchetti, T. Ranzani, G. Gerboni, I. De Falco, C. Laschi, and A. Menciassi. Stiff-flop surgical manipulator: Mechanical design and experimental characterization of the single module. In 2013 IEEE/RSJ International Conference on Intelligent Robots and Systems, pages 3576-3581, Nov 2013. doi: 10.1109/IROS.2013. 6696866.

[6] Wyatt Felt and C David Remy. Smart braid: Air muscles that measure force and displacement. In Intelligent Robots and Systems (IROS 2014), 2014 IEEE/RSJ International Conference on, pages 2821-2826. IEEE, 2014.

[7] Wyatt Felt, Khai Yi Chin, and C. David Remy. Contraction sensing with smart braid McKibben muscles. Mechatronics, IEEE/ASME Transactions on, PP(99):11, 2015. ISSN 1083-4435. doi: 10.1109/TMECH.2015. 2493782.

[8] Wyatt Felt, Michelle Suen, and C David Remy. Sensing the motion of bellows through changes in mutual inductance. In Intelligent Robots and Systems (IROS 2016), 2016 IEEE/RSJ International Conference on. IEEE, 2016.

[9] Wyatt Felt, Khai Yi Chin, and C. David. Remy. Smart braid feedback for the closed-loop control of soft robotic systems. Soft Robotics (In Press), 2017.

[10] Festo. Bionic handling assistant, 2010. URL http://www. festo.com/cms/en_corp/9655_10218.htm.

[11] Festo. BionicMotionRobot, Mar 2017. URL https://www. festo.com/group/en/cms/12747.htm

[12] I Gaiser, R Wiegand, O Ivlev, A Andres, H Breitwieser, $\mathrm{S}$ Schulz, and $\mathrm{G}$ Bretthauer. Compliant robotics and automation with flexible fluidic actuators and inflatable structures. 2012.

[13] M. T. Gillespie, C. M. Best, and M. D. Killpack. Simultaneous position and stiffness control for an inflatable soft robot. In 2016 IEEE International Conference on Robotics and Automation (ICRA), pages 1095-1101, May
2016. doi: 10.1109/ICRA.2016.7487240.

[14] Teichert Systemtechnik GmbH. Innovative cable-likeshape-sensor, Accessed April 2017. URL http://www. tst-inno.de/en/SAC.html

[15] G. Granosik and J. Borenstein. Integrated joint actuator for serpentine robots. Mechatronics, IEEE/ASME Transactions on, 10(5):473-481, Oct 2005. ISSN 1083-4435. doi: 10.1109/TMECH.2005.856222.

[16] Michael D Grissom, Vilas Chitrakaran, Dustin Dienno, Matthew Csencits, Michael Pritts, Bryan Jones, William McMahan, Darren Dawson, Chris Rahn, and Ian Walker. Design and experimental testing of the octarm soft robot manipulator. In Defense and Security Symposium, pages 62301F-62301F. International Society for Optics and Photonics, 2006.

[17] Christoph Ledermann, Julien Mintenbeck, Yitao Ding, Hendrikje Pauer, and Heinz Wörn. Closed-loop control of a flexible instrument using an integrated FBG-based shape sensor. In International Conference on Advanced Technology \& Sciences (ICAT'15), 2015.

[18] A.D. Marchese, K. Komorowski, C.D. Onal, and D. Rus. Design and control of a soft and continuously deformable 2D robotic manipulation system. In Robotics and Automation (ICRA), 2014 IEEE International Conference on, pages 2189-2196, May 2014. doi: 10.1109/ICRA. 2014.6907161.

[19] Franz Ernst Neumann. Allgemeine gesetze der induzierten elektrischen strme. pages 1-87, 1845.

[20] Jones Franklin D. Horton Holbrook L. Ryffel Henry H. Oberg, Erik. Machinery's Handbook (29th Edition) \& Guide to Machinery's Handbook. Industrial Press, 2012. ISBN 978-0-8311-2901-9.

[21] Oberhauser, Chris at Texas Instruments. LDC Sensor Design, 2014. URL http://www.ti.com/lit/an/snoa930/ snoa930.pdf.

[22] Tatsuyuki Ochi. A positioning system for mobile robots using symmetrical rotating laser beams. Advanced Robotics, 4(3):217-222, 1989.

[23] R. S. Penning, J. Jung, N. J. Ferrier, and M. R. Zinn. An evaluation of closed-loop control options for continuum manipulators. In Robotics and Automation (ICRA), 2012 IEEE International Conference on, pages 5392-5397, 2012. doi: 10.1109/ICRA.2012.6224735.

[24] Gianluca Rizzello, David Naso, Alexander York, and Stefan Seelecke. A self-sensing approach for dielectric elastomer actuators based on online estimation algorithms. IEEE/ASME Transactions on Mechatronics, 2016.

[25] Sina Sareh, Yohan Noh, Min Li, Tommaso Ranzani, Hongbin Liu, and Kaspar Althoefer. Macrobend optical sensing for pose measurement in soft robot arms. Smart Materials and Structures, 24(12):125024, 2015.

[26] StretchSense. Stretchsense evaluation kit datasheet, June 2015. URL http://stretchsense.com/wp-content/uploads/ 2015/06/Stretch-Sensor-Datasheet.pdf

[27] Texas Instruments. Application Report: Using Multiple Sensors with LDC1000, 2014. URL http://www.ti.com/ 
lit/an/snoa924/snoa924.pdf

[28] C. To, T.L. Hellebrekers, and Yong-Lae Park. Highly stretchable optical sensors for pressure, strain, and curvature measurement. In Intelligent Robots and Systems (IROS), 2015 IEEE/RSJ International Conference on, pages 5898-5903, Sept 2015. doi: 10.1109/IROS. 2015.7354215.

[29] Ran Xu, Ali Asadian, Anish S Naidu, and Rajni V Patel. Position control of concentric-tube continuum robots using a modified jacobian-based approach. In Robotics and Automation (ICRA), 2013 IEEE International Conference on, pages 5813-5818. IEEE, 2013.

[30] Ali Yassin, Youssef Nasser, Mariette Awad, Ahmed AlDubai, Ran Liu, Chau Yuen, and Ronald Raulefs. Recent advances in indoor localization: A survey on theoretical approaches and applications. IEEE Communications Surveys \& Tutorials, 2016.

[31] Alan Yates and Jeremy Selan. Positional tracking systems and methods, May 12 2016. US Patent 20,160,131,761.

[32] Huichan Zhao, Kevin O'Brien, Shuo Li, and Robert F. Shepherd. Optoelectronically innervated soft prosthetic hand via stretchable optical waveguides. Science Robotics, 1(1), 2016. doi: 10.1126/scirobotics. aai7529. URL http://robotics.sciencemag.org/content/1/ 1/eaai7529. 